\title{
Ocorrência de desvios posturais em escolares do ensino público fundamental de Jaguariúna, São Paulo
}

\author{
Occurrence of postural deviations in children of a school of Jaguariúna, São Paulo, Brazil
}

\author{
Camila Isabel S. Santos ${ }^{1}$, Alexandre Baccili N. Cunha², Viviane Pereira Braga², Ivete Alonso B. Saad ${ }^{3}$, Maria Ângela G. O. Ribeiro ${ }^{4}$ \\ Patrícia Blau M. Conti ${ }^{5}$, Telma D. Oberg ${ }^{6}$
}

\section{RESUMO}

Objetivo: Avaliar a postura de escolares do ensino público fundamental e diferenciar as alterações que fazem parte do crescimento normal das compensatórias.

Métodos: Trata-se de um estudo analítico, descritivo, de corte transversal, no qual se realizou avaliação postural em escolares de primeira a quarta série de uma escola pública da cidade de Jaguariúna, situada no interior do Estado de São Paulo. Cada aluno foi avaliado nos planos coronal-anterior, coronal-posterior e sagital, utilizando-se o método de Kendall como referência de alinhamento postural normal.

Resultados: A avaliação postural de 247 escolares (131 masculinos e 116 femininos) identificou a seguinte incidência de alterações: desnível $(50,2 \%)$ e protrusão de ombro $(39,7 \%)$, escápula alada (40,5\%), aumento do ângulo valgo de joelho (29,6\%), inclinação $(21,5 \%)$ e anteroversão pélvica (19\%), hiperextensão de joelho (19\%), rotação de fêmur $(12,9 \%)$, protrusão $(11,7 \%)$ e inclinação cervical $(15,4 \%)$, cifose torácica $(9,7 \%)$ e hiperlordose lombar $(26,3 \%)$.

Conclusões: Houve elevada incidência de escápula alada, desnível e protrusão de ombro, aumento do ângulo valgo de joelho e hiperlordose lombar, consideradas normais durante o desenvolvimento da criança. As inclinações e a protrusão cervical, alterações que necessitam de intervenção precoce, também foram identificadas.
Palavras-chave: incidência; postura; estudantes.

\section{ABSTRACT}

Objective: Evaluate the posture of students from a public school in Brazil and to identify the differences between normal deviation during growth and compensatory alterations.

Methods: Students from first to fourth grade of a public school in the city of Jaguariúna, São Paulo, Brazil, were evaluated. The students were positioned at sagital anterior and posterior coronal planes for postural assessment. Kendall points were used as the normal reference.

Results: 247 students were evaluated, 131 boys and 116 girls, and the main postural deviations found were: shoulder unbalance $(50.2 \%)$, protracted shoulder $(39.7 \%)$, abducted scapula $(40.5 \%)$, knock-knee $(29.6 \%)$, pelvic unbalance $(21.5 \%)$, pelvic anteversion (19\%), knee hyperextension $(19 \%)$, medial rotation of hip (12.9\%), protracted cervical (11.7\%), head tilt (15.4\%), thoracic hyperkyphosis (9.7\%) and lumbar hyperlordosis (26.3\%).

Conclusions: A high incidence of postural alterations was detected in school children. Some of the postural alterations, such as abducted scapula, unbalance and protraction of the shoulders, knock-knee and lumbar hiperlordosis, normally occur and they are naturally corrected during growth. However, some postural problems, such as protraction and inclination of

${ }^{5}$ Supervisora dos Cursos de Especialização e Aprimoramento em Fisioterapia Pediátrica da FCM-Unicamp, Campinas, SP, Brasil

${ }^{6}$ Professora Doutora, coordenadora do Curso de Graduação da FAJ e da Especialização em Fisioterapia Aplicada a Neurologia Adulta da FCMUnicamp, Campinas, SP, Brasil

Endereço para correspondência:

Camila Isabel S. Santos

Rua Décio de Almeida Filho, 73 - Barão Geraldo

CEP 13084-710 - Campinas/SP

E-mail: cacaiss@yahoo.com.br

Recebido em: 15/5/08

Aprovado em: 19/10/08 
the cervical spine, were also prevalent and they require early intervention.

Key-words: incidence; posture; students.

\section{Introdução}

A postura pode ser definida como a posição ou a atitude do corpo em disposição estática ou o arranjo harmônico das partes corporais a situações dinâmicas ${ }^{(1)}$. Uma boa postura é resultado da capacidade que os ligamentos, cápsulas e tônus muscular têm de suportar o corpo ereto, permitindo sua permanência em uma mesma posição por períodos prolongados, sem desconforto e com baixo consumo energético ${ }^{(2)}$.

Na postura padrão, a coluna apresenta curvaturas normais e os ossos dos membros inferiores ficam em alinhamento ideal para a sustentação de peso. A posição neutra da pelve conduz ao bom alinhamento do abdome, do tronco e dos membros inferiores. O tórax e a coluna superior se posicionam de forma que a função ideal dos órgãos respiratórios seja favorecida. A cabeça fica ereta, bem equilibrada, minimizando a sobrecarga sobre a musculatura cervical ${ }^{(2)}$.

Em crianças, variações posturais são comumente encontradas no período do crescimento e desenvolvimento, sendo decorrentes dos vários ajustes, adaptações e mudanças corporais e psicossociais que marcam essa fase ${ }^{(3)}$. Penha et al alertam para o fato de que a postura da criança e do adolescente pode ser afetada por vários fatores intrínsecos e extrínsecos, como hereditariedade, ambiente e condições físicas nas quais o indivíduo vive, bem como por fatores emocionais, socioeconômicos e por alterações consequentes do crescimento e desenvolvimento humano ${ }^{(3)}$.

Hábitos posturais inadequados são transmitidos de geração a geração. Inicialmente, as crianças copiam as atitudes adotadas pelos adultos, sejam elas corretas ou não, e, posteriormente, as incorporam ou modificam ${ }^{(4)}$. Atualmente, observa-se um aumento significativo na incidência de problemas posturais em crianças de todo o mundo, sendo as causas mais comuns a má postura durante as aulas, o uso incorreto de mochila escolar, a utilização de calçados inadequados, o sedentarismo e a obesidade ${ }^{(3,5-8)}$.

Para Ascher ${ }^{(9)}$, o comportamento postural da criança durante os primeiros anos escolares é o grande responsável pelos vícios posturais adquiridos, levando-se em consideração a evolução da sua postura ereta, suas condições anatômicas, sua coluna vertebral e as relações estabelecidas com o meio social em que vive ${ }^{(9)}$. Com uma avaliação minuciosa da postura é possível identificar alterações localizadas em diversos segmentos corporais como: protrusão de ombros, aumento da cifose torácica, inclinações e rotações pélvicas, aumento do ângulo valgo de joelho. Essas alterações, geralmente, trazem consequências prejudiciais à função de sustentação e mobilidade e, portanto, seu diagnóstico precoce permite uma intervenção eficiente, principalmente tratando-se de um sistema músculoesquelético complacente, como o da criança.

Sendo assim, este estudo tem o objetivo de avaliar a postura de escolares de primeira a quarta série do ensino público fundamental, identificar a incidência de alterações que fazem parte do crescimento normal e as que necessitam de intervenção precoce.

\section{Método}

Neste estudo analítico, descritivo e de corte transversal, participaram todos os alunos de primeira a quarta série do ensino fundamental, regularmente matriculados na escola municipal Prefeito Joaquim Pires Sobrinho, localizada na cidade de Jaguariúna, interior de São Paulo. Os pais e responsáveis pelos alunos incluídos no estudo foram notificados sobre o procedimento de avaliação postural e consentiram.

Trata-se de um projeto de prevenção, promoção e proteção à saúde, envolvendo uma parceria entre a prefeitura local e a faculdade como parte de um projeto social vinculado ao Serviço de Saúde da cidade de Jaguariúna. O presente estudo apresenta os resultados de tal projeto, previamente aprovado pelas instituições envolvidas.

As avaliações posturais foram realizadas por alunos do último ano do curso de graduação em Fisioterapia da Faculdade de Jaguariúna, no período da manhã durante oito meses. Os avaliadores foram previamente treinados e supervisionados pela profissional responsável pelo estágio em Fisioterapia Preventiva e Saúde Comunitária.

Individualmente, cada aluno foi avaliado sobre uma superfície plana, descalço, com os pés separados em $7,5 \mathrm{~cm}$, com traje de banho. Como padrão postural de referência, utilizou-se o padrão de pontos proposto por Kendall(2), visualizado em três planos: (1) no plano coronal-anterior, observou-se a inclinação e a rotação cervical, a elevação de ombro e inclinação pélvica, a rotação de tronco, fêmur e tíbia, o alinhamento patelar e a angulação do joelho; (2) no plano coronal-posterior, observou-se o alinhamento da escápula e da coluna, a prega glútea e a poplítea, o posicionamento dos pés e a confirmação de algumas alterações vistas no plano coronal-anterior; (3) no plano sagital, observou-se a retificação e protrusão cervical, a protrusão de ombro, o alinhamento torácico e lombar, as rotações pélvicas e a hiperextensão de 


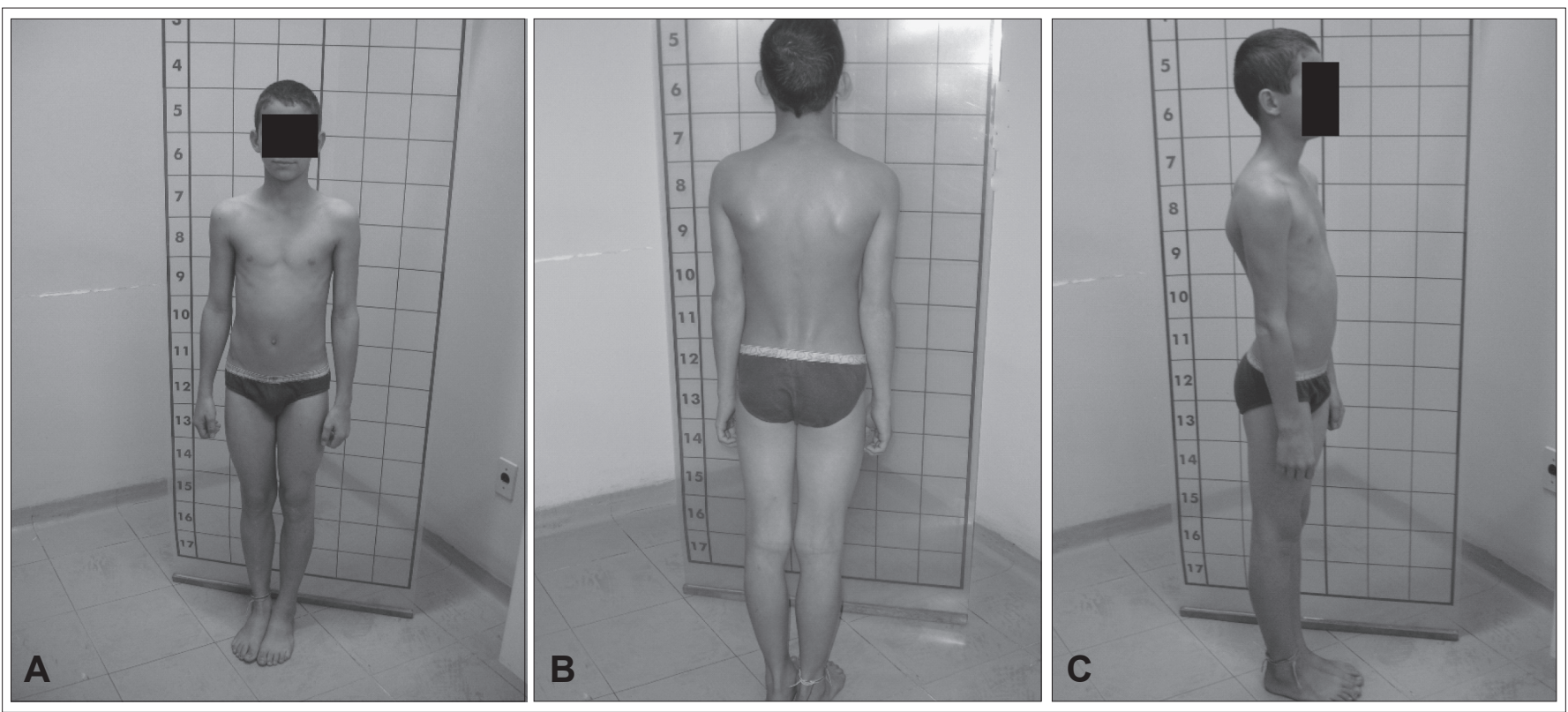

Figura 1 - Avaliação postural: (A) planos coronal-anterior, (B) coronal-posterior, (C) sagital.

Tabela 1 - Número absoluto de alunos por grupo e distribuição por sexo

\begin{tabular}{lccc}
\hline Idade & \multicolumn{2}{c}{ Sexo } & Total de alunos \\
\hline Grupo 1 & 28 & 20 & 48 \\
Grupo 2 & 26 & 28 & 54 \\
Grupo 3 & 24 & 28 & 52 \\
Grupo 4 & 32 & 23 & 55 \\
Grupo 5 & 21 & 17 & 38 \\
Total & $\mathbf{1 3 1}$ & $\mathbf{1 1 6}$ & $\mathbf{2 4 7}$ \\
\hline
\end{tabular}

Tabela 2 - Frequência das alterações posturais

\begin{tabular}{lc}
\hline Alterações posturais & Frequência (\%) \\
\hline Desnível de ombro & $124(50,2)$ \\
Escápula alada & $100(40,6)$ \\
Protusão de ombro & $98(39,7)$ \\
Valgo de joelho & $73(29,6)$ \\
Hiperlordose lombar & $65(26,3)$ \\
Inclinação pélvica & $53(21,4)$ \\
Antero-versão pélvica & $47(19,0)$ \\
Hiperextensão de joelho & $47(19,0)$ \\
Escoliose & $39(15,7)$ \\
Inclinação cervical & $38(15,4)$ \\
Rotação de fêmur & $32(12,9)$ \\
Protusão cervical & $29(11,7)$ \\
Cifose torácica & $24(9,7)$ \\
Total & $\mathbf{2 4 7}(\mathbf{1 0 0})$ \\
\hline
\end{tabular}

joelho (Figura 1). Os dados da avaliação postural foram registrados em uma ficha padronizada, contendo a identificação do aluno, sua data de nascimento, série escolar e as alterações observadas em cada um dos três planos.

A análise dos dados foi feita por meio da estatística descritiva, com o software SPSS versão 7.5. Os alunos foram analisados de acordo com a faixa etária e distribuídos em cinco grupos: Grupo 1 (seis anos e seis meses a sete anos e seis meses), Grupo 2 (sete anos e sete meses a oito anos e seis meses); Grupo 3 (oito anos e sete meses a nove anos e seis meses), Grupo 4 (nove anos e sete meses a dez anos e seis meses) e Grupo 5 (dez anos e sete meses a 13 anos e seis meses). Tal agrupamento foi realizado para diferenciar as alterações consideradas normais para a idade daquelas patológicas, bem como comparar a incidência de desvios por grupo etário.

\section{Resultados}

Foram analisados 247 escolares, sendo 131 do sexo masculino e 116 do feminino, com idade média de oito anos e 11 meses, variando entre seis anos e seis meses a 12 anos e cinco meses. A distribuição do número de escolares em cada grupo por sexo é apresentada na Tabela 1.

$\mathrm{Na}$ análise da frequência dos desvios posturais pelo número total de alunos, independentemente da idade, observouse que $50,2 \%$ dos alunos apresentaram desnível de ombro e 40,6\% escápulas aladas. Apenas $9,7 \%$ mostraram cifose torácica (Tabela 2). 
Tabela 3 - Frequência de alterações posturais por grupo etário

\begin{tabular}{lccccc}
\hline Alterações posturais & Grupo 1 (\%) & Grupo 2 (\%) & Grupo 3 (\%) & Grupo 4 (\%) & Grupo 5 (\%) \\
\hline Protrusão cervical & 2,0 & 5,5 & 7,7 & 18,2 & 24,9 \\
Inclinação cervical & 35,4 & 24,0 & 10,6 & 7,2 & 0,0 \\
Desnível de ombro & 29,1 & 64,8 & 57,8 & 49,1 & 50,0 \\
Protrusão de ombro & 20,8 & 37,0 & 50,5 & 40,5 & 49,9 \\
Escápula alada & 31,3 & 31,5 & 55,8 & 45,5 & 38,8 \\
Cifose torácica & 10,8 & 0,0 & 19,5 & 12,7 & 5,3 \\
Hiperlordose lombar & 12,5 & 14,8 & 25,0 & 40,0 & 39,1 \\
Ântero-versão pélvica & 16,7 & 31,5 & 6,8 & 21,8 & 18,4 \\
Inclinação pélvica & 33,3 & 31,5 & 18,3 & 11,0 & 13,1 \\
Rotação de fêmur & 4,6 & 20,4 & 23,6 & 5,4 & 10,5 \\
Hiperextensão de joelho & 0,0 & 26,5 & 30,8 & 19,5 & 18,4 \\
Valgo de joelho & 30,5 & 27,8 & 21,8 & 34,6 & 33,1 \\
\hline
\end{tabular}

De acordo com a faixa etária (Tabela 3), evidenciou-se que alterações como desnível e protrusão de ombro e escápula alada estiveram presentes em todos os grupos etários, com frequência acima de $20 \%$. O desnível de ombro foi identificado em $64 \%$ das crianças do Grupo 2, acima de sete anos; já a protrusão de ombro e a escápula alada foram mais frequentes no Grupo 3.

$\mathrm{Na}$ análise da presença de um ou mais desvios associados, observou-se que em cerca de $20 \%$ das 247 crianças estavam presentes três ou quatro alterações. Apenas $2 \%$ dos alunos não apresentaram nenhuma alteração postural (Tabela 4).

\section{Discussão}

Durante o crescimento, as proporções do corpo atingem gradualmente a forma do adulto. $\mathrm{O}$ crescimento é maior no início da infância, diminui até o começo da adolescência e volta a acelerar durante esse período. O crescimento das várias epífises também é variável. No membro superior ele é mais rápido no ombro e no punho e, no membro inferior, na epífise do joelho ${ }^{(10)}$.

Apesar da desaceleração na velocidade de crescimento ósseo com os anos, vários aspectos relacionados às posturas e hábitos das crianças passam a ser determinantes para o desenvolvimento muscular e esquelético. Principalmente no período de sete a 12 anos de idade, quando ocorre a busca do equilíbrio às novas proporções do corpo, evidenciam-se as transformações posturais ${ }^{(5)}$.

O período crítico ocorre quando a criança inicia o aprendizado e a escrita. Os padrões adequados e inadequados de postura e movimento começam a ser determinados na infância, são praticados na adolescência e logo se tornam habituais. Em longo prazo, os padrões inadequados culminam na aceleração do processo de degeneração do sistema
Tabela 4 - Frequência de crianças que apresentaram uma ou mais alterações posturais associadas

\begin{tabular}{lc}
\hline Alterações posturais & Frequência (\%) \\
\hline 1 alteração & $27(11,0)$ \\
2 alterações & $40(16,0)$ \\
3 alterações & $54(22,0)$ \\
4 alterações & $56(22,7)$ \\
5 alterações & $36(14,5)$ \\
6 alterações & $20(8,0)$ \\
7 alterações & $5(2,0)$ \\
8 alterações & $3(1,2)$ \\
9 alterações & $1(0,5)$ \\
Sem alterações & $5(2,0)$ \\
Total & $\mathbf{2 4 7}(\mathbf{1 0 0 , 0 )}$ \\
\hline
\end{tabular}

músculo-esquelético, o que pode trazer uma predisposição às afecções da coluna vertebral no adulto, manifestadas por quadros álgicos ${ }^{(11)}$.

As alterações capazes de levar a compensações patológicas, como a escoliose, a cifose e a hiperlordose lombar, têm grande incidência em alunos do $1^{\circ}$ grau escolar ${ }^{(12-14)}$. Padrões posturais inadequados assumidos durante a fase escolar podem se tornar permanentes na idade adulta, caso não haja intervenção durante a fase de crescimento e estruturação óssea ${ }^{(15)}$.

Este estudo, que avaliou a postura de 247 escolares, identificou um índice elevado de alguns desníveis posturais, com distribuição distinta de acordo com a faixa etária. Mesmo realizado por alunos do último ano de graduação previamente treinados e com supervisão de uma profissional fisioterapeuta, a sensibilidade para a identificação das alterações foi alta, considerando-se os resultados apresentados.

A alteração mais frequente foi o desnível de ombros $(50,2 \%)$. Alguns autores associam a presença dessa assimetria ao fato de o indivíduo ser destro ou canhoto, por promover 
hipertrofia muscular mais acentuada no lado dominante, o que pode causar uma elevação de tal ombro(3,5).

Segundo Sacco et al ${ }^{(16)}$, a assimetria do ombro está relacionada ao suporte de mochilas escolares de maneira inadequada, lembrando que ajustes posturais e ações compensatórias surgem diante da aplicação dessas cargas assimétricas. $\mathrm{O}$ modo como cada indivíduo carrega a sua carga pode ser determinado por fatores como o peso, o tamanho e a forma do utensílio escolar, o tempo de transporte, o terreno, o clima, a característica e a constituição física do indivíduo. Os desequilíbrios posturais gerados nessas situações são agravados pelo fato de o peso carregado ser frequentemente desproporcional ao peso do próprio corpo e pelo uso inadequado da mochila, como no caso do apoio em um único ombro.

Essa sobrecarga acarreta depressão de um dos ombros, podendo trazer consequências para a musculatura local. Ao deprimir o ombro, músculos como o elevador da escápula, fibras superiores do músculo trapézio e romboides estão sendo alongados de maneira abrupta, mas a tendência é que trabalhem no sentido de modular tal depressão para que se protejam as articulações da cintura escapular por meio da contração muscular. Prova disso é a tendência a elevar o ombro durante a contração muscular excessiva causada pelo uso prolongado de mochilas escolares de peso inadequado. Tais músculos estariam realizando um trabalho excêntrico durante a depressão e, posteriormente, um trabalho isométrico para estabilizar o ombro.

Também foi frequente, na população estudada, a presença de escápula alada, identificada em 40,5\% dos alunos. Apesar de ter sido a segunda alteração postural mais observada, sua incidência foi inferior à do trabalho de Ferronato ${ }^{(15)}$, no qual a alteração esteve presente em $100 \%$ dos alunos avaliados com idade entre sete e 14 anos. No entanto, os resultados deste estudo foram próximos aos de Penha et $\mathrm{al}^{(3)}$, que identificou a mesma alteração em $64,7 \%$ de meninas de sete a dez anos de idade. Já no estudo realizado em Novo Hamburgo ${ }^{(17)}$, a presença de escápula alada incidiu em $80,5 \%$ da amostra de 154 alunas entre sete e 17 anos, sendo a alteração mais observada, seguida de protrusão cervical $(66,2 \%)$ e do ombro $(47,4 \%)$.

Com relação à protrusão de ombro, terceira alteração mais frequente, existe relativo consenso em considerá-la uma alteração fisiológica, parte do desenvolvimento muscular infantil. Para Tanaka e Farah ${ }^{(18)}$, a protrusão de ombro está associada à abdução escapular e é impulsionada pela ação de grupos musculares. A ação predominante do serrátil ante- rior e do peitoral maior sobre o romboide, trapézio médio e superior proporcionam uma rotação medial do úmero e uma tração anterior do complexo do ombro. Pini ${ }^{(19)}$ relata que essa protrusão tende a diminuir progressivamente após os dez anos de idade, afirmando que tal alteração faz parte do desenvolvimento normal da criança.

Também considerada uma alteração fisiológica, o aumento do ângulo valgo de joelho foi identificado em 29,6\% dos alunos avaliados. Todd e Patrick ${ }^{(20)}$ relatam que o recémnascido inicia seu ciclo vital com rotação medial de tíbia e rotação lateral de fêmur, caracterizando um valgo de joelho. Os autores afirmam que, após o nascimento, esse processo inicia-se lentamente para que, entre oito e dez anos, a rotação de tíbia esteja presente entre $15^{\circ}$ e $25^{\circ}$ de rotação externa, diminuindo a angulação do valgo de joelho. Lapierre ${ }^{(21)} \mathrm{e}$ Asher $^{(9)}$ afirmam que cerca de $80 \%$ das crianças de uma população geral apresentam joelho valgo/varo durante a fase do desenvolvimento e que tal alteração aparece dos três aos seis anos de idade, desaparecendo em seguida. Os resultados do presente estudo mostraram incidência dessas alterações em faixa etária superior (9-13 anos), em concordância com os resultados de Oliveira et $a l^{(22)}$.

A incidência de $26,3 \%$ de crianças com hiperlordose lombar neste estudo pode ser considerada baixa, se comparada ao resultado de $58 \%$ de casos identificados por Penha el $\mathrm{al}^{(3)}$. Segundo Detsch e Candotti ${ }^{(17)}$, até os nove anos, a presença de hiperlordose lombar é normal no desenvolvimento infantil, uma vez que não há estabilidade postural, o que gera necessidade de busca pelo equilíbrio corporal por protrusão abdominal e aumento da inclinação pélvica anterior. Para alguns autores, alterações fisiológicas como a hiperlordose lombar, a ântero-versão pélvica e a hiperextensão de joelho têm causas comuns. Dentre essas, cita-se a fraqueza do retoabdominal e dos paravertebrais, sendo o trabalho dessa musculatura do complexo abdominal mais efetivo a partir dos dez ou 12 anos de vida ${ }^{(10,23-25)}$.

Em relação às alterações compensatórias, mecanismos da biomecânica para manter a linha de gravidade no centro do corpo e promover um menor gasto energético, a inclinação pélvica foi observada em $21,5 \%$ dos alunos. A diferença no comprimento do membro inferior, a alteração unilateral da fáscia plantar, a contratura na musculatura adutora ou abdutora podem ser fatores desencadeantes desse mecanismo compensatório ${ }^{(2,16,26,27)}$.

Outras alterações identificadas neste estudo (inclinação cervical, rotação medial de fêmur, protrusão cervical e 
cifose torácica) são descritas como mecanismos compensatórios da postura, considerando-se a centralização da linha de gravidade ${ }^{(28)}$. Esses mecanismos compensatórios ocorrem em decorrência de desequilíbrios musculares, explicados pela diferença de força e flexibilidade entre grupos musculares que atuam sobre uma mesma articulação, isto é, quando determinado grupo muscular se apresenta mais forte e mais tensionado do que seu respectivo antagonista ${ }^{(29)}$.

Os desequilíbrios ocorrem pela promoção de um desalinhamento que altera o posicionamento de composições ósseas. Essa alteração é decorrente de encurtamentos musculares ou de sobrecargas excessivas em articulações, ligamentos e outras estruturas, podendo causar lesões agudas ou crônico-degenerativas ${ }^{(2)}$.

Alguns grupos musculares apresentam predisposição natural ao encurtamento. Embora não exista uma explicação para isso, acredita-se que há relação com a posição fetal. Dentre os músculos que sabidamente tendem ao encurtamento, destacam-se: eretores espinhais, quadrado lombar, tensor da fáscia lata, piriforme, reto femoral, gastrocnêmio e sóleo, peitoral maior, trapézio superior, elevador da escápula, esternocleidomastoideo e escalenos; seus antagonistas diretos tendem, por sua vez, ao estiramento ${ }^{(29)}$.

O processo de instalação de um desequilíbrio muscular em geral não é perceptível ao indivíduo até que suas consequências comecem a se manifestar, em geral na forma de quadros álgicos e deformidades. Levando-se em consideração a estrutura de cadeias musculares que compõem o corpo humano, o processo será seguido de uma série de compensações locais e à distância, transformando o problema inicial em um complexo processo que necessitará de reabilitação postural ${ }^{(29)}$.

Um estudo realizado nos Estados Unidos revelou que, numa população de 20 milhões de incapacitados, 8,4 milhões eram devido a doenças da coluna ${ }^{(30)}$. Ao relacionar o ambiente escolar com a postura, percebe-se que os problemas têm origens diversas: causas ergonômicas, como as encontradas no transporte do material escolar; arquitetura desfavorável do imóvel; disposições e proporções inadequadas do mobiliário, as quais, provavelmente, serão responsáveis pela manutenção, aquisição ou agravamento de hábitos posturais inapropriados ${ }^{(30)}$.

O índice de escoliose deste trabalho foi de $15,7 \%$, correspondendo a valores abaixo dos encontrados na literatura. Em um estudo realizado com escolares de sete a 12 anos em uma escola pública de São Paulo ${ }^{(29)}$, constatou-se a prevalência de hiperlordose lombar em $73,8 \%$, escoliose em $23,8 \%$ e hipercifose torácica em $9,5 \%$. Penha et al avaliaram um grupo de
132 crianças do sexo feminino entre sete e dez anos de idade com fotografias em planos frontal e sagital e observaram a presença de escoliose em 36\% das crianças com sete anos, $45 \%$ nas de oito anos, $52 \%$ nas de nove anos e $48 \%$ nas de dez $\operatorname{anos}^{(3)}$. Ferriani et al ${ }^{(31)}$ investigaram as alterações posturais em 378 escolares na faixa etária dos seis aos 14 anos e detectaram $23,5 \%$ de casos suspeitos de escoliose, mensurados pelo teste de Adams. Mangueira ${ }^{(32)}$ observou que os desvios da coluna vertebral foram os principais achados em uma amostra com 166 crianças e adolescentes entre 11 e 16 anos. A presença de hipercifose torácica foi de $34,9 \%$, seguida de escoliose $(27,7 \%)$ e hiperlordose lombar $(17,5 \%)$. Os resultados obtidos em relação à protrusão cervical $(11,7 \%)$ foram inferiores aos os encontrados nos estudos de Mangueira ${ }^{(32)}$ (47\%) e de Detsch e Candotti ${ }^{(17)}$ (66\%).

Weis e Muller ${ }^{(33)}$ afirmam que o alto índice de crianças com alterações na coluna cervical indica que muitos alunos em idade escolar não apresentam postura adequada da cabeça durante as atividades, principalmente em sala de aula, o que promove acentuada curva na região cervical, prejudicando o equilíbrio corporal.

Por tratar-se de um trabalho de iniciação científica de um grupo de alunos do nível de graduação, alguns aspectos metodológicos foram limitados e devem ser considerados em estudos futuros. A avaliação postural foi feita por mais de um avaliador, o protocolo utilizado para avaliação apresenta deficiências e não se aplicou um questionário prévio para possível identificação de fatores pré e pós-natais com potencial para influenciar o crescimento e o desenvolvimento das crianças (intercorrências pré e pós-parto, pé torto congênito, hálux valgo infantil) ${ }^{(17)}$. Apesar dessas limitações, identificaram-se resultados importantes.

Rosa $\mathrm{Neto}^{(34)}$ refere que a avaliação postural feita nas escolas, quando realizada por profissionais interessados em melhorar a saúde escolar, pode contribuir muito para a sociedade, evitando enfermidades futuras na coluna vertebral, melhorando o rendimento do aluno e promovendo o seu bem-estar físico e emocional. Este estudo epidemiológico atingiu o objetivo de identificar alterações posturais presentes em escolares do ensino público, sejam essas fisiológicas, como o desnível e a protrusão de ombro, a escápula alada, a hiperlordose lombar e a hiperextensão de joelho, ou alterações compensatórias, como a inclinação e a protrusão cervical, a rotação medial de fêmur e a cifose torácica. As compensações merecem atenção especial e precoce pela possibilidade de instalação, progressão e agravamento durante a adolescência e a vida adulta, assim como a persistência das alterações fisiológicas pode comprometer a postura nessas fases. 
Fica expressa, aqui, a necessidade de futuros estudos que graduem as variações consideradas normais durante o desenvolvimento para que possa ser feita precocemente a identificação e a necessária intervenção diante do diagnóstico de uma alteração fora dos padrões de normalidade.

\section{Referências bibliográficas}

1. Lehmkuhl LD, Smith LK. Cinesiologia Clínica de Brunnstron. $5^{\mathrm{a}}$ ed. São Paulo, Barueri: Manole; 1997.

2. Kendall FP, McCreary EK, Provance PG. Músculos: provas e funções. $4^{\mathrm{a}}$ ed. São Paulo: Manole; 1995.

3. Penha PJ, João SM, Casarotto RA, Amino CJ, Penteado DC. Postural assessment of girls between 7 and 10 years of age. Clinics 2005;60:9-16

4. Hoebel EA, Frost EL. Antropologia cultural e social. $2^{\mathrm{a}}$ ed. São Paulo: Cultrix; 1976.

5. Rodrigues LF, Fernandes M, Barros JW, Shimano AC, Moreira FBR, Gonçalves FF et al. Utilização da técnica de Mire para detectar alterações posturais. Rev Fisioter Univ São Paulo 2003;10:16-23.

6. Lincoln TL, Suen PW. Common rotational variations in children. J Am Acad Orthop Surg 2003;11:312-20.

7. Mac-Thiong JM, Berthonnaud E, Dimar JR 2nd, Betz RR, Labelle H. Sagittal alignment of the spine and pelvis during growth. Spine 2004;29:1642-7.

8. Pietrobelli A, Faith MS, Allison DB, Gallagher D, Chiumello G, Heymsfield SB. Body mass index as a measure of adiposity among children and adolescents: a validation study. J Pediatr 1998;132:204-10.

9. Asher C. Variações da postura na criança. São Paulo: Manole; 1976.

10. Hebert SK. Ortopedia para pediatras. São Paulo: Artmed; 2004.

11. Resende FL, Borsoe AM. Investigation of postural problems in 6-8 year old children from a school in São José dos Campos, São Paulo. Rev Paul Pediatr 2006;24:42-6.

12. Ingelmark BE. The influence of different forms of physical education upon the posture in childhood. FIEP Bull 1953;3:18-26.

13. Clarke HH. Joint and body range of movement. Phys Fitness Res Dig 1975;5:1-22

14. Brighetti V, Bankoff AD. Survey of the incidence the of postural kyphosis and fallen shoulders in students from 1st to 4th levels. Rev Bras Cienc Esporte 1986;7:93-7.

15. Ferronatto $A$, Candotti $C T$, Silveira RP. A incidência de alterações de equilíbrio estático da cintura escapular em crianças entre 7 a 14 anos. Movimento 1998;9:24-30.

16. Sacco IC, Melo MC, Rojas GB, Naki IK, Burgi K, Silveira LT et al. Biomechanical and kinesiological study of postures trough digital photographs: cases report. R Bras Ci e Mov 2003;11:25-33.

17. Detsch C, Candotti CT. A incidência de desvios posturais em meninas de 6 a 17 anos da cidade de Novo Hamburgo. Movimento 2001;7:43-56.

18. Tanaka C, Farah EA. Anatomia funcional das cadeias musculares. São Paulo: Ícone; 1997.

19. Pini MC. Fisiologia esportiva. Rio de Janeiro: Guanabara Koogan; 1978.

20. Lincoln TL, Suen PW. Common rotational variations in children. J Am Acad Orthop Surg 2003;11:312-20.

\section{Agradecimentos}

Agradecemos à aluna do curso de Aprimoramento em Fisioterapia Pediátrica, Renata Tiemi Okuro, que colaborou com a organização deste manuscrito para a submissão.

21. Lapierre A. Reeducação física: cinesiologia, reeducação postural, reeducação psicomotora - Vol. II. 6a ed. São Paulo: Manole; 1982.

22. Oliveira C, Nagassaki E, Rosa J, Gerardi JF, Matsuoca KT, Bertoldi LF et al Avaliação e orientação postural em escolares de 7-12 anos do Colégio Estadual Jardim Piza, Roseira [monograph on the Internet]. Curso de avaliação postural - secção artigos; 2003 [cited 2008 Dec 20] Available from: http://www. ccs.uel.br/olhomagico/peepin98/Gim16.html.

23. Bienfait M. Os desequilíbrios estáticos: filosofia, patologia e tratamento fisioterápico. $4^{\mathrm{a}}$ ed. São Paulo: Summus; 1995.

24. Bankoff AD, Campelo TS, Ciol P, Zamai CA. Postura e equilíbrio corporal: um estudo das relações existentes. Movim Percep 2006;6:55-70.

25. Nordin M, Frankel VH. Biomecânica básica do sistema musculoesquelético. $3^{\text {a }}$ ed. Rio de Janeiro: Guanabara Koogan; 2003.

26. Magee DJ. Avaliação musculoesquelética. $4^{a}$ ed. São Paulo: Manole; 2005.

27. Wells JP, Hyler-Both DL, Danley TD, Wallace GH. Biomechanics of growth and development in the healthy human infant: a pilot study. J Am Osteopath Assoc 2002;102:313-9.

28. Efisioterapia.net [homepage on the Internet]. Rodrigues BG. Desequilíbrios musculares: fortalecimento, alongamento e relaxamento com o método Pilates [monograph on the Internet]. Madri: Escola de Osteopatia de Madri; 2007 [cited 2008 Aug 18]. Available from: http://www.efisioterapia.net/articulos/leer228.php.

29. Jassi FJ, Pastre CM [homepage on the Internet]. Alterações posturais na coluna vertebral em escolares do ensino fundamental da cidade de Adamantina SP [monograph on the Internet]. Faculdades Adamantinenses Integradas; 2004 [cited 2008 Sep 20] Avaliable from: http://www.fai.com.br/ fisio/resumos2/13.doc.

30. Martelli RC, Traebert J. Descriptive study of backbone postural changes in 10 to 16 year-old schoolchildren. Tangará-SC, Brazil, 2004. Rev Bras Epidemiol 2006;9:87-93

31. Ferriani MG, Cano MA, Candido GT, Kanchina AS. Levantamento epidemiológico dos escolares portadores de escoliose da rede pública de ensino de $1^{\circ}$ grau no município de Ribeirão Preto. Rev Eletr Enfermagem [serial on the Internet]. 2000;2(1) [cited 2008 May 28]. Available from: http://www.fen. ufg.br/revista/revista2_1/28.05.2008/Levanta.html

32. Mangueira JO. Prevalência de desvios da coluna vertebral ao exame físico em estudantes de 11 a 16 anos em uma escola do bairro Sinhá Sabóia em Sobral - CE [tese de mestrado]. Sobral (CE): Universidade Estadual Vale do Acaraú; 2004.

33. Weis GF, Müller U. Dinamizando a prática do exame biométrico: cuidados necessários a uma postura correta. Rev Professor 1994;40:36-44.

34. Rosa Neto F. Avaliação postural em escolares de $1^{\mathrm{a}}$ a $4^{\mathrm{a}}$ Série do $1^{\circ}$ grau. Rev Bras Cienc Movim 1991;5:7-11. 